\title{
Expression of cellCAM-105 in the apical surface of rat uterine epithelium is controlled by ovarian steroid hormones
}

\author{
P. C. Svalander* $\ddagger$, P. Odin $\uparrow \S$, B. O. Nilsson* and B. Öbrink $\dagger$ \\ ${ }^{*}$ Department of Human Anatomy, Uppsala University, Uppsala, Sweden; and \\ $\uparrow$ Department of Medical Cell Biology, Karolinska Institute, Stockholm, Sweden
}

\begin{abstract}
Summary. Affinity-purified antibodies to cellCAM-105, an adhesive cell surface glycoprotein, were used in immunohistochemical investigations of rat uteri at various functional stages: (i) the oestrous, pro-oestrous, metoestrous, and dioestrous stages of the oestrous cycle, (ii) Days 1-8 of normal pregnancy, (iii) delayed implantation, (iv) $18 \mathrm{~h}$ after oestrogen reactivation from delay of implantation, and (v) juvenile rats, and normal ovariectomized adults, respectively, before and after experimental injection of progesterone and/or oestrogen. CellCAM-105 was present in the apical zones of the luminal and glandular epithelium cells in a stage-specific and hormone-dependent manner.

The results indicate that: (1) steroid hormones are essential for the expression of cellCAM-105 in the uterine epithelial cells; (2) progesterone induces cellCAM-105 expression in the glandular epithelium, and oestrogen induces cellCAM-105 expression in the luminal epithelium; (3) progesterone induces down-regulation of cellCAM-105 from the surface of the uterine luminal epithelium of juvenile rats; (4) cellCAM-105 is absent in the luminal epithelial cells but present in the glandular epithelial cells of the rat uterus at the time of blastocyst implantation.
\end{abstract}

Keywords: cellCAM-105; rat; uterus; oestrous cycle; implantation; progesterone; oestrogen

\section{Introduction}

In the rat, the early stage of blastocyst implantation involves cellular adhesion between the trophectoderm and the luminal epithelium of the uterus, following which the trophoblast penetrates the epithelium and invades the endometrial stroma (Schlafke \& Enders, 1975). A prerequisite for successful implantation is that the endometrium has been sensitized appropriately by ovarian steroid hormones, and this takes place during the reproductive cycle (Casimiri \& Psychoyos, 1981; Psychoyos, 1986). Progesterone must prime the endometrium for some $48 \mathrm{~h}$ before oestrogen can induce a short phase of endometrial blastocyst receptivity (Psychoyos \& Martel, 1985).

That cell adhesion involves interactions between specific cell surface molecules is well established (see reviews by Edelman, 1986; Öbrink, 1986). Previous attempts to characterize cell surface molecules involved in the acquisition of endometrial blastocyst receptivity in the rabbit and mouse have revealed that alterations occur in the glycoprotein composition of the luminal surface of the uterus (Lampelo et al., 1985; Chávez \& Anderson, 1985; Anderson et al., 1986). Although there are several studies indicating that implantation-specific molecular changes occur in the uterine luminal

\footnotetext{
†Present address: Department of Obstetrics and Gynaecology, Karolinska Hospital, Box 60500, S-104 01 Stockholm, Sweden. §Present address: Department of Neurology, University Hospital, Lund, Sweden.
} 
epithelium, at the molecular level data are still lacking and the molecular nature of the membrane interactions at implantation is unclear at present.

CellCAM-105 is a membrane-integrated, cell-surface glycoprotein, originally identified as being involved in intercellular adhesion of rat hepatocytes in vitro (Ocklind \& Öbrink, 1982). CellCAM105 , isolated from the liver of the rat, consists of two highly glycosylated, structurally similar peptide chains of apparent $M_{\mathrm{r}} 105000$ and 110000 , respectively (Odin et al., 1986). The molecule is expressed in several simple and stratified epithelia, platelets and granulocytes of the adult rat (Odin \& Öbrink, 1987; Odin et al., 1988). CellCAM-105 has been found on the surface of early, preimplantation blastocysts and of blastocysts in delay of implantation although the molecule disappears from the abembryonal trophectoderm of adhesive, implantation blastocysts (Svalander et al., 1987). This suggested that the disappearance of cellCAM-105 from the trophectoderm may be involved in the implantation process. Further, it seemed probable that the presence of cellCAM105 on the trophectoderm was controlled by ovarian steroid hormones, acting either directly on the blastocyst or indirectly via hormone-induced alterations in the endometrium.

Previous studies have demonstrated that cellCAM-105 is present in uterine epithelia of the rat (Odin, 1987; Odin \& Öbrink, 1987; Odin et al., 1988) and it was suspected that the expression of the molecule was influenced by the hormonal fluctuations occurring during the reproductive cycle. This prompted us to perform a detailed investigation of the rat uterus, with special emphasis on the possibility that ovarian steroid hormones might control the expression of cellCAM-105.

The objective of this study was to investigate the appearance and distribution of cellCAM105 in different hormonal states of the uterus occurring during the oestrous cycle and in early pregnancy. The influence of progesterone and oestrogen on the uterine expression of cellCAM-105 was also investigated.

\section{Materials and Methods}

Antibodies. Affinity-purified rabbit IgG antibodies (anti-cellCAM antisera 7, 8, 9 and 10) against cellCAM-105 were used. The antisera were produced and affinity-purified as described previously (Odin et al., 1986). The specificity of the antibodies was determined by immunoprecipitation of ${ }^{125}$ I-labelled, detergent-solubilized rat hepatocytes followed by two-dimensional electrophoresis and autoradiography as described previously (Odin et al., 1986). All four anti-cellCAM IgG preparations were equally specific and reacted exclusively with the A-and B-chains of cellCAM-105 (Odin et al., 1988).

Animals. Virgin females, aged 2 months (adult) or 4 weeks (juvenile), of the Sprague-Dawley strain (Alab, Stockholm, Sweden) were used in the experiments. The animals were housed under standard conditions at the AnimalCare Department, Biomedical Centre, Uppsala, in a temperature-controlled room with a 12-h light-dark cycle, and fed ad libitum with a diet of R3 rat pellets (Ewos AB, Södertälje, Sweden).

Determination of stage of oestrus. Four stages of the regular 4-day oestrous cycle of the rat were determined by examining vaginal smears according to the criteria described by Rogers \& Gannon (1981). Exfoliative vaginal smears were obtained by probing with a spatula and the material recovered was smeared on glass slides and examined by light microscopy. Uteri of 3 rats in each of the stages of oestrus were retained for immunohistochemical analysis of cellCAM-105 (see below).

Normal pregnancy. For the induction of pregnancy, females were caged with males overnight. The following morning when a vaginal plug was found was designated Day 1 of pregnancy. Uteri of Days 1-8 of pregnancy (parturition usually occurs at Day 21) were removed for immunohistochemical analysis of cellCAM-105 (see below), and 24 rats, 3 for each of the pregnancy Days 1-8, were included in this set of experiments.

Experimental delay of implantation. Delay of implantation was induced as described previously (Svalander et al., 1987). Briefly, the rats were ovariectomized at Day 3 of pregnancy and injected every 5 th day with $1 \mathrm{mg}$ medroxyprogesterone acetate, ready-to-use immersion containing $50 \mathrm{mg}$ hormone $/ \mathrm{ml}$ (Depo-Provera; Upjohn Co., Puurs, Belgium). Reactivation from delay was induced by injecting $0 \cdot 1 \mu \mathrm{g}$ oestradiol- $17 \beta$ (Sigma Chemical Co., St Louis, MO, USA) dissolved in polypropylene glycol at a concentration of $1 \mu \mathrm{g} / \mathrm{ml}$. The adhesion stage of implantation is reached $18 \mathrm{~h}$ after oestrogen injection (Bergström, 1978). Three rats at the delay stage and three rats at the 
adhesion stage were included in this set of experiments. The uteri were prepared for immunohistochemical analysis of cellCAM-105 (see below).

Treatment of ovariectomized rats with ovarian steroid hormones. Virgin adult rats were ovariectomized and 10 days later given subcutaneous injections of progesterone and oestrogen, separately or in combination. This set of experiments included 4 rats for each of the following treatments: Group (a) - 1 dose of progesterone ( $1 \mathrm{mg})$, and removal of the uteri 4 days later; Group (b)-l dose of progesterone $(1 \mathrm{mg})$ and 4 days later 2 doses of oestrogen $(0 \cdot 1 \mu \mathrm{g}$ each) at 24-h intervals, and removal of the uteri $24 \mathrm{~h}$ later; Group (c) - 2 doses of oestrogen $(0 \cdot 1 \mu \mathrm{g} \mathrm{each})$ at 24 -h invervals, and removal of the uteri $24 \mathrm{~h}$ later; Group (d) -5 doses of oestrogen $(0.1 \mu \mathrm{g}$ each) at $24 \mathrm{~h}$ intervals, and removal of the uteri $24 \mathrm{~h}$ later; Group (e)-control group, without treatment and hysterectomy 10 days after ovariectomy. All uteri were prepared for immunohistochemical analysis of cellCAM-105 (see below).

Treatment of juvenile rats with ovarian steroid hormones. Juvenile female rats were given subcutaneous injections of progesterone and/or oestrogen. This set of experiments consisted of identical experimental groups of hormone treatments as with the ovariectomized adult rats (see above). In the control group, the uteri were removed without previous treatment. Each group consisted of 2 rats. All uteri were prepared for immunohistochemical analysis of cellCAM-105 (see below).

Tissue processing. The rats were given a lethal intraperitoneal dose of pentobarbitone sodium (ACO, Solna, Sweden). The uterine horns were excised and washed quickly in Dulbecco's phosphate-buffered saline (PBS-D; $\left.140 \mathrm{~mm}-\mathrm{NaCl} / 2.7 \mathrm{mM}-\mathrm{KCl} / 8.1 \mathrm{~mm}-\mathrm{Na}_{2} \mathrm{HPO}_{4} / 1.5 \mathrm{~mm}-\mathrm{KH}_{2} \mathrm{PO}_{4} / 0.68 \mathrm{mM}-\mathrm{CaCl}_{2} / 0.49 \mathrm{mM}-\mathrm{MgCl}_{2} / \mathrm{pH} 7.4\right)$, cut transversely into pieces, $4-5 \mathrm{~mm}$ long, and incubated in a fixative of $4 \%(\mathrm{w} / \mathrm{w})$ freshly prepared paraformaldehyde/PBS-D at $4^{\circ} \mathrm{C}$ for $16 \mathrm{~h}$. After fixation, the specimens were incubated in PBS-D overnight and dehydrated by sequential incubation in two changes each lasting $10 \mathrm{~h}$ of $70 \%$ ethanol at $4^{\circ} \mathrm{C}$, two changes each lasting $1.5 \mathrm{~h} \mathrm{of} 95 \%$ ethanol at $20^{\circ} \mathrm{C}$, three changes each lasting $1 \mathrm{~h}$ of $99.5 \%$ ethanol at $20^{\circ} \mathrm{C}$, three changes each lasting $1 \mathrm{~h}$ of xylene at $20^{\circ} \mathrm{C}$ and embedded in paraffin wax $\left(16-20 \mathrm{~h}\right.$ at $\left.60^{\circ} \mathrm{C}\right)$.

Paraffin-wax blocks of each uterine horn were prepared for transverse sectioning. Sections of $7 \mu \mathrm{m}$ were cut, mounted and warm-fixed $\left(30^{\circ} \mathrm{C}\right)$ on chrome-alum-coated glass slides. The slides were stored at $20^{\circ} \mathrm{C}$ until used.

$A B C$ staining procedure. CellCAM-105 was visualized in the rat uterus by the avidin-biotin-peroxidase complex (ABC) method, which is based on a primary antibody, a biotinylated secondary antibody, and a complex of avidin and biotinylated horseradish peroxidase which is allowed to react with a chromogenic substrate (Hsu et al., 1981; Bourne, 1983).

The slides, each containing $8-10$ transverse sections, $7 \mu \mathrm{m}$ thick, of a specified uterine horn were deparaffinized and rehydrated at room temperature by sequential incubation in two changes each lasting $5 \mathrm{~h}$ of xylene, two changes each lasting $5 \mathrm{~min}$ of the following: $99 \cdot 5 \%$ ethanol, $95 \%$ ethanol, $70 \%$ ethanol and water. Ultrapure, distilled water was used unless otherwise stated.

Endogeneous peroxidase activity was inhibited routinely by incubating the rehydrated sections in $3 \%$ hydrogen peroxide in tap water for $20 \mathrm{~min}$. Non-specific antibody adsorption was reduced by incubation in $1 \%$ normal pig serum (NSS) and $0.1 \%$ bovine serum albumin (BSA) in PBS-D for $3 \mathrm{~h}$ in a moist chamber.

After the blocking treatment, the sections were incubated in $75 \mu \mathrm{l}$ of affinity-purified anti-cellCAM IgG antibodies or preimmune-IgG in $1 \% \mathrm{NSS}$ and $0.1 \%$ BSA in PBS-D for $60 \mathrm{~min}$ at room temperature in a moist chamber. The antibody was tested at concentrations of $3 \cdot 5,17$ and $34 \mu \mathrm{g} / \mathrm{ml}$. The slides were washed in PBS-D three times each lasting $10 \mathrm{~min}$. The excess liquid was then blotted from the slides and the sections were incubated for $20 \mathrm{~min}$ at room temperature in a moist chamber in $75 \mu \mathrm{l}$ biotinylated pig anti-rabbit immunoglobulin (Dakopatts $\mathrm{A} / \mathrm{S}$, Glostrup, Denmark), diluted $1: 200$ in $1 \%$ NSS and $0.1 \%$ BSA in PBS-D. Washing was repeated and the sections incubated for $30 \mathrm{~min}$ at room temperature in a moist chamber in $75 \mu \mathrm{ABC}$ reagent (Vectastain; Vector Laboratories Inc., Burlingame, CA, USA). The ABC reagent was prepared according to the manufacturer's instructions. The slides were then washed in PBS-D three times each lasting $10 \mathrm{~min}$ and incubated for $15 \mathrm{~min}$ in a substrate solution prepared immediately before use by mixing $10 \mathrm{mg}$ 3-amino-9-ethylcarbazole (Sigma) dissolved in $6 \mathrm{ml}$ dimethylsulphoxide (DMSO) with $50 \mathrm{ml} 20 \mathrm{mM}$-sodium acetate buffer, pH 5.5, and adding $4 \mu 130 \% \mathrm{H}_{2} \mathrm{O}_{2}$. After washing in tap water, the sections were counterstained with Mayer's haematoxylin, then washed in tap water and mounted in Kaiser's glyceringelatin. The sections were examined by light microscopy immediately after mounting and then stored in the dark at room temperature for subsequent re-examination. The results were based on the examination of immunohistochemical staining of at least 80 random, transverse sections of each uterine specimen. The examination was performed independently by two investigators.

Immunohistochemical controls. All samples were checked for non-specific antibody adsorption, endogenous peroxidase activity, and endogenous avidin-binding activity. Parallel sections were incubated for ABC staining (1) with an equal amount of the corresponding preimmune-IgG antibody, isolated by affinity chromatography on protein ASepharose (Ocklind \& Öbrink, 1982), and (2) without any Ig. Endogenous avidin-binding activity was also tested for by incubating parallel sections in $0.1 \%$ avidin followed by $0.01 \%$ biotin (Wood \& Warnke, 1981); the ABC procedure was then begun with incubation in ABC-reagent.

Microscopy. Stained tissue sections were viewed in a Nikon Labophot light microscope and photographed on Kodak Ektachrome 160 ASA Tungsten colour film. 


\section{Results}

The results of the immunohistochemical localization of cellCAM-105 during various hormonal influences in the rat uterus are summarized in Table 1. The changes in the concentrations of progestagens and oestrogens during the oestrous cycle and early pregnancy in the rat are presented schematically in Fig. 1.

Table 1. CellCAM-105 staining (negative $(-)$ and positive

$(+))$ in the rat uterus

\begin{tabular}{|c|c|c|}
\hline & $\begin{array}{l}\text { Luminal } \\
\text { epithelium }\end{array}$ & $\begin{array}{l}\text { Glandular } \\
\text { epithelium }\end{array}$ \\
\hline \multicolumn{3}{|l|}{ Endogenous hormonal influence } \\
\hline \multicolumn{3}{|l|}{ Oestrous cycle } \\
\hline Metoestrus & - & - \\
\hline Dioestrus & - & + \\
\hline Pro-oestrus & + & - \\
\hline Oestrus (ovulation) & + & - \\
\hline \multicolumn{3}{|l|}{ Pregnancy (day) } \\
\hline 1 & + & + \\
\hline 2 & + & - \\
\hline 3 & - & - \\
\hline 4 & - & - \\
\hline 5 (implantation) & - & + \\
\hline 6 & - & + \\
\hline 7 & _. & + \\
\hline 8 & - & + \\
\hline \multicolumn{3}{|l|}{ Exogenous hormonal influence } \\
\hline \multicolumn{3}{|l|}{ Facultative delay } \\
\hline Delay of implantation $(\mathrm{P})$ & - & + \\
\hline $18 \mathrm{~h}$ after reactivation $(\mathrm{P}+\mathrm{E})$ & - & + \\
\hline \multicolumn{3}{|l|}{ Ovariectomized adults } \\
\hline Without treatment & - & - \\
\hline$P(\times 1)$ & - & + \\
\hline$E(\times 2)$ & - & - \\
\hline$E(\times 5)$ & + & - \\
\hline$P(\times 1)+E(\times 2)$ & - & + \\
\hline \multicolumn{3}{|l|}{ Juveniles } \\
\hline Without treatment & + & + \\
\hline$P(\times 1)$ & - & + \\
\hline$E(\times 2)$ & + & + \\
\hline$E(\times 5)$ & + & + \\
\hline$P(\times 1)+E(\times 2)$ & - & + \\
\hline
\end{tabular}

\section{Endogenous hormonal influence}

CellCAM-105 during the oestrous cycle. The epithelial expression of cellCAM-105 varied during the cyclic changes of the uterus (Fig. 2a-d). CellCAM-staining was observed in the uterine luminal epithelium only at the pro-oestrous (Fig. 2a) and oestrous stages (Fig. 2b). The uterine glandular epithelium was simultaneously cellCAM-negative. The uterine glandular epithelium exhibited cellCAM staining only in the dioestrous stage (Fig. $2 \mathrm{~d}$ ). 


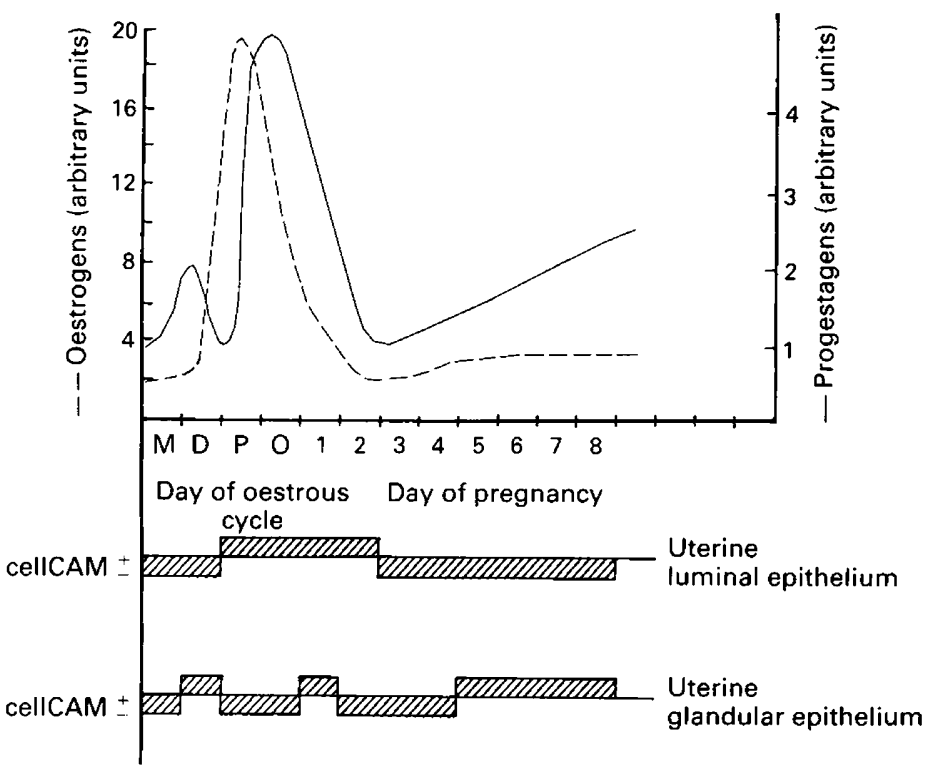

Fig. 1. Schematic diagram of changes in the concentrations of oestrogens and progestagens during the oestrous cycle and early pregnancy in the rat (constructed after Hashimoto et al., 1968; Shaikh, 1971; Surani, 1977). The appearance of cellCAM-105 in the uterine luminal and glandular epithelia is indicated for comparison. $\mathrm{M}=$ metoestrus; $\mathrm{D}=$ dioestrus; $\mathbf{P}=$ prooestrus; $\mathrm{O}=$ oestrus.

CellCAM-105 during early pregnancy. The epithelial expression of cellCAM-105 varied during Days 1-8 of pregnancy (Table 1).

CellCAM-105 was detected in the uterine luminal epithelium at Days 1 and 2 of pregnancy. From Day 3 onwards, however, no cellCAM staining was observed in the uterine luminal epithelium (Table 1).

The uterine glandular epithelium exhibited cellCAM staining at Day 1 of pregnancy, followed by an absence of cellCAM staining during Days 2-4. However, cellCAM-105 reappeared and became detectable in the glandular epithelium at Day 5 of pregnancy (the day of implantation), and remained until Day 8, which was the last stage studied (Table 1).

Immunohistochemistry of serially sectioned Day-8 implantation sites revealed no cellCAM staining of the embryo or of the trophoblast (Fig. 2f).

\section{Exogenous hormonal influence}

CellCAM-105 in delay of implantation and after reactivation from delay. At delay of implantation, and at $18 \mathrm{~h}$ after oestrogen reactivation from delay (the adhesion stage), cellCAM-105 was detected in the glandular epithelium only (Table 1).

CellCAM-105 in ovariectomized adult rats after treatment with ovarian steroid hormones. In the absence of ovarian steroid hormones, the uterine luminal and glandular epithelia of ovariectomized rats were devoid of cellCAM staining (Table 1; Fig. 2g). Administration of progesterone, alone or in combination with two doses of oestrogen, resulted in the appearance of cellCAM-105 in the glandular epithelium, but not in the luminal epithelium (Table 1; Fig. 2h). CellCAM-105 was detected in the luminal epithelium only after 5 consecutive daily doses of oestrogen, but was not detected in the glandular epithelium (Table 1). 

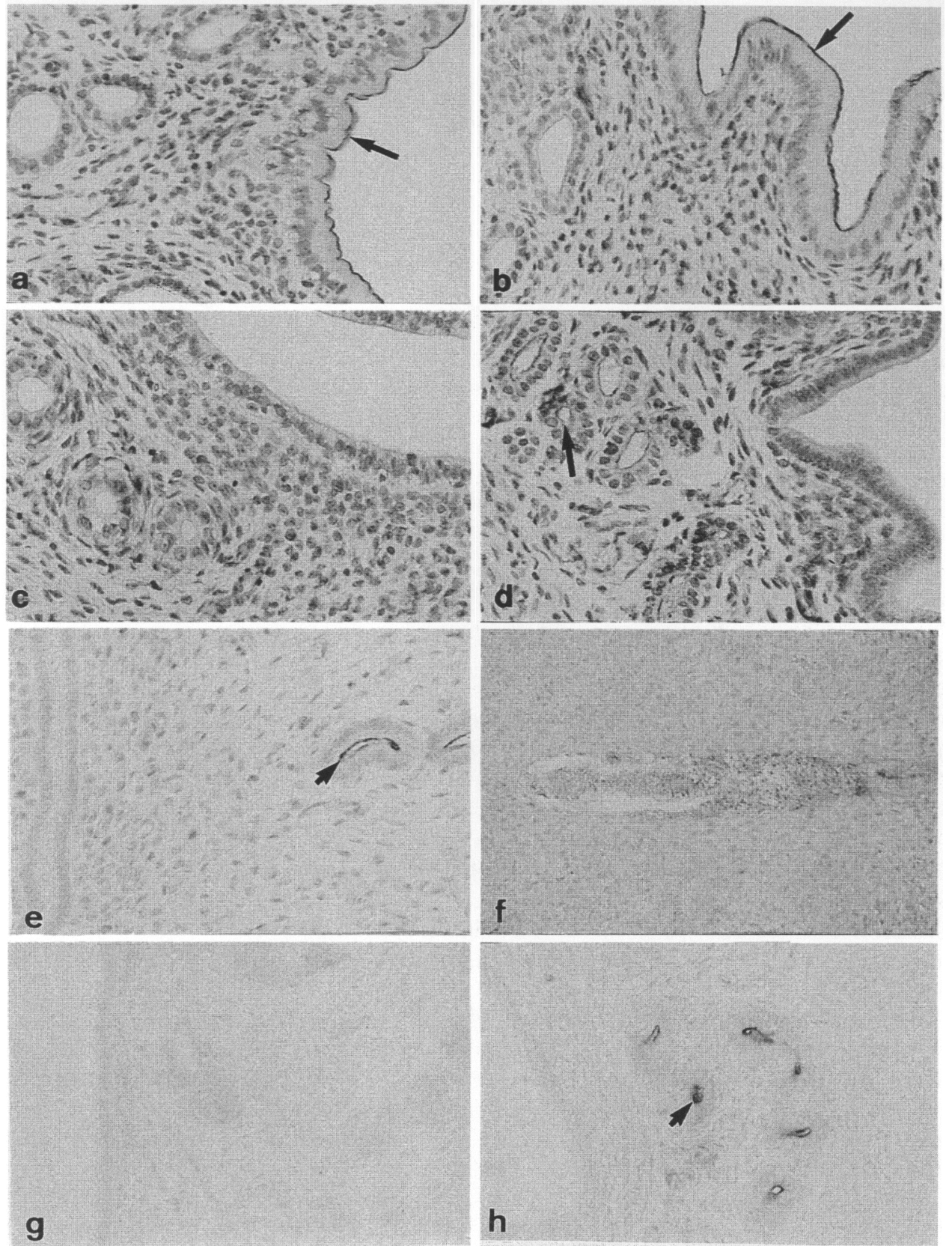

Fig. 2. Immunolocalization of cellCAM-105 in the uteri of rats in the oestrous cycle (a-d), normal pregnancy $(\mathrm{e}, \mathrm{f})$ and after ovariectomy $(\mathrm{g}, \mathrm{h})$. (a) Pro-oestrus: the apical zone of the luminal epithelium exhibits cellCAM staining (arrow) $\times 1100$. (b) Oestrus: the staining pattern (arrow) is similar to that of the pro-oestrous stage. $\times 1100$. (c) Metoestrus: cellCAM is undetectable in the uterus. $\times 1100$. (d) Dioestrus: the apical part of the glandular epithelium exhibits staining for cellCAM (arrow) whereas the luminal epithelium is negative. $\times 1100$. (e) Day 7 of normal pregnancy: the uterine glandular epithelium is cellCAM-positive (arrow) whereas the luminal epithelium is negative. $\times 400$. (f) Day-8 implanting blastocyst: all embryonic cells and the luminal epithelium are devoid of staining (the ectoplacental cone trophoblast is located to the right), the glandular epithelium is not shown but had cellCAM-staining. $\times 400$. (g) Uterus of an ovariectomized rat without hormone treatment: staining for cellCAM is absent. $\times 400$. (h) Uterus of an ovariectomized rat treated with one dose $(1 \mathrm{mg})$ of progesterone: the glandular epithelium is stained (arrow) whereas the luminal epithelium is devoid of staining. $\times 400$. 
CellCAM-105 in juvenile rats after treatment with ovarian steroid hormones. CellCAM staining occurred both in the uterine luminal and glandular epithelium of untreated juvenile rats and this pattern of staining was unaffected by injection of oestrogen. However, the injection of progesterone, alone or in combination with 2 doses of oestrogen, resulted in the disappearance of cellCAM-105 from the luminal epithelium, but not from the glandular epithelium (Table 1).

Immunohistochemical controls. All controls were negative.

\section{Discussion}

Immunochemical and immunohistochemical studies of cellCAM-105 have revealed that this molecule is preferentially expressed by stationary, terminally differentiated, or differentiating cells (Odin et al., 1988; Odin \& Öbrink, 1988). We have proposed that the molecule may be involved in the organization of the structure of the normal liver and of certain epithelia into their mature patterns (Odin et al., 1988). Results obtained from several different experimental systems have indicated that a decrease in concentration of cellCAM-105 on the cell surface may be correlated to increased cellular motility and proliferation. For example, in liver regenerating after partial hepatectomy, the concentration of cellCAM-105 decreased transiently to about one-third of that of normal liver (Odin \& Öbrink, 1986). In transplantable rat hepatocellular carcinomas, cellCAM-105 is absent or chemically modified (Hixson et al., 1985).

Biochemical identification of cellCAM-105 in the uterus of the rat has been conducted previously by a quantitative radioimmunoassay, and by SDS-polyacrylamide gel electrophoresis and immunoblotting with affinity-purified anti-cellCAM IgG (Odin, 1987; Odin \& Öbrink, 1987; Odin et al., 1988). Four different anti-cellCAM antibodies yielded identical patterns: a single, rather broad band appeared with a mean apparent molecular weight of 105000 (96000-117000) under non-reducing conditions. This apparent $M_{\mathrm{r}}$ was slightly higher than that of cellCAM-105 in the liver, indicating minor macromolecular differences between liver-cell CAM and uterus-cell CAM. The differences may reside in the carbohydrate and/or the peptide part of the molecule and might reflect differences in functional properties.

In the present work, we found that the expression of cellCAM-105 in the uterus changed dynamically during the reproductive cycle. Furthermore, the pattern of expression differed in the luminal and the glandular epithelia, respectively. These fluctuations could be mimicked by injection of ovarian steroid hormones. The luminal epithelium expressed cellCAM-105 in response to oestrogen, whereas the glandular epithelium expressed cellCAM-105 in response to progesterone. Progesterone was also found to induce a down-regulation of cellCAM-105 from the uterine luminal epithelium.

Accordingly, it seems likely that the expression of this molecule is influenced by the fluctuating concentrations of progesterone and oestrogen occurring during the reproductive cycle. CellCAM105 appeared in the glandular epithelium in correlation with the two peak levels of progesterone, namely at dioestrus and around Day 1 of pregnancy (Hashimoto et al., 1968), at both of which oestrogen is low. The reappearance of cellCAM-105 in the glandular epithelium at the time of implantation may be due to the rise in progesterone or to a synergistic effect of oestrogen and progesterone. In the luminal epithelium the cellCAM-105 expression correlated with the peak of oestrogen during pro-oestrus and oestrus, and during the first day of pregnancy. The disappearance of cellCAM-105 from the luminal epithelium correlated with high progesterone concentrations during met- and dioestrus, and from Day 3 of pregnancy onwards.

Previous studies in the rat have shown that differences in the response to ovarian hormones exist in the uterine luminal and glandular epithelia (for review, see Finn \& Porter, 1975). For instance, epithelial proliferation, and an increase in the length and number of microvilli in the luminal epithelium are signs of oestrogen action, but not of progesterone action. Under physiological conditions, oestrogen and progesterone act together either concurrently or consecutively and exert a variety of cellular effects, including increased synthesis and translation of mRNA (O'Malley 
\& Means, 1974). In the endometrial cells of the rat, progesterone and oestrogen bind to separate cytoplasmic receptor molecules and affect different nuclear target sites. Furthermore, progesterone antagonizes oestrogen in the endometrial cells by reducing the amount of cytoplasmic oestrogen receptor (Hsueh et al., 1975). Thus, the action of oestrogen and progesterone on the endometrium during the reproductive cycle is diverse and complex. Our results demonstrate for the first time differential expression of a specific molecule in the luminal and glandular epithelia as a function of oestrogen and progesterone action.

During the interaction between the blastocyst and uterine epithelia at implantation, the expression of cellCAM-105 changes. During delay of implantation, cellCAM-105 is present on the trophectoderm but not on the luminal surface of the uterus, but when implantation begins cellCAM-105 persists only on the polar trophoblast, i.e. the portion of the trophectoderm overlying the inner cell mass. Later, at Day 8 of pregnancy, cellCAM-105 is absent from all embryonic cells, as determined by investigating serial sections of implanted blastocysts. On the other hand, the glandular epithelium expresses cellCAM-105 both during delay and at implantation. The decrease in the concentration of cellCAM-105 on the surfaces of both the trophectoderm and the uterine luminal epithelium may be a prerequisite for implantation.

Blastocysts are incapable of implantation into a uterus which has not been properly sensitized by ovarian steroid hormones (Cowell, 1969), but are capable of implantation at extrauterine sites (Fawcett, 1950; Kirby, 1963a, b) regardless of the hormonal environment. It is possible that the apical surface of the non-receptive uterine luminal epithelium expresses molecules which prevent the blastocyst from invading the uterus. It therefore seems relevant to assume that ovarian steroid hormones induce specific molecular changes in the luminal surface of the uterus to allow the trophoblast to adhere to and then to penetrate and invade the endometrium. The changes in expression of cellCAM-105 in the luminal epithelium could be part of such a mechanism. The disappearance of cellCAM-105 may result in fewer cross-linked cell surfaces which might facilitate cellular dissociation and motility necessary for the penetration and invasion of the uterine luminal epithelium by the trophectodermal cells at implantation.

We thank Ms Barbro Einarsson, Ms Marianne Ljungkvist, Ms Karin Obrist and Ms Birgitta Wärmegård for excellent technical assistance; and Dr Bill Mair for linguistic revision of the manuscript.

The study was supported financially by The Swedish Medical Research Council (project nos. 05200, 6686 and 00070), The Swedish Cancer Foundation (project no. 1389), Gustaf V:s 80-års fond, The "Expressen" Prenatal Research Foundation, Magn. Bergvalls Stiftelse, and the Lars Hierta Foundation. Depo-Provero was kindly donated by Upjohn AB, Sweden.

\section{References}

Anderson, T.L., Olson, G.E.\&Hoffman, L.H. (1986) Stagespecific alterations in the apical membrane glycoproteins of endometrial epithelial cells related to implantation in rabbits. Biol. Reprod. 34, 701-720.

Bergström, S. (1978) Experimentally delayed implantation. In Methods in Mammalian Reproduction, pp. 419-435. Ed. J. C. Daniel, Jr. Academic Press, New York.

Bourne, J.A. (1983) Handbook of Immunoperoxidase Staining Methods. Dako Corporation, Santa Barbara.

Casimiri, V. \& Psychoyos, A. (1981) Embryoendometrial relationships during implantation. In The Endometrium, pp. 63-80. Eds J. DeBrux, R. Mortel \& J. P. Gautray. Plenum Press, New York.

Chávez, D.J. \& Anderson, T.L. (1985) The glycocalyx of the mouse uterine luminal epithelium during estrus, early pregnancy, the peri-implantation period, and delayed implantation. 1. Acquisition of Ricinus Communis 1 binding sites during pregnancy. Biol. Reprod. 32, 1135-1142.

Cowell, T.P. (1969) Implantation and development of mouse eggs transferred to the uteri of nonprogestational mice. J. Reprod. Fert. 19, 239-245.

Edelman, G.M. (1986) Cell adhesion molecules in the regulation of animal form and tissue pattern. Ann. Rev. Cell Biol. 2, 81-116.

Fawcett, D.W. (1950) The development of mouse ova under the capsule of the kidney. Anat. Rec. 108, 71-91.

Finn, C.A. \& Porter, D.G. (1975) The Uterus. Elek Science, London.

Hashimoto, I., Henricks, D.M., Anderson, L.L. \& Melampy, R.M. (1968) Progesterone and pregn-4-en$20 \beta$-ol-3-one in ovarian venous blood during various 
reproductive states in the rat. Endocrinology 82, 333-341.

Hixson, D.C., McEntire, K.D. \& Öbrink, B. (1985) Alterations in the expression of a hepatocyte cell adhesion molecule by transplantable rat hepatocellular carcinomas. Cancer Res. 45, 3742-3749.

Hsu, S.M., Raine, L. \& Fanger, H. (1981) Use of avidinbiotin-peroxidase complex $(\mathrm{ABC})$ in immunoperoxidase techniques: a comparison between $\mathrm{ABC}$ and unlabeled antibody (PAP) procedures. J. Histochem. Cytochem. 29, 577-580.

Hsueh, A.J.W., Peck, E.J., Jr \& Clark, J.H. (1975) Progesterone antagonism of the oestrogen receptor and oestrogen-induced uterine growth. Nature, Lond. 254, 337-339.

Kirby, D.R.S. (1963a) Development of the mouse blastocyst transplanted to the spleen. J. Reprod. Fert. 5, $1-12$.

Kirby, D.R.S. (1963b) The development of mouse blastocysts transplanted to the scrotal and cryptorchid testis. J. Anat. 97, 119-130.

Lampelo S.A., Ricketts, A.P. \& Bullock, D.W. (1985) Purification of rabbit endometrial plasma membranes from receptive and non-receptive uteri. $J$. Reprod. Fert. 75, 475484.

Öbrink, B. (1986) Epithelial cell adhesion molecules. Expl Cell Res. 163, 1-21.

Ocklind, C. \& Öbrink, B. (1982) Intercellular adhesion of rat hepatocytes. Identification of a cell surface glycoprotein involved in the initial adhesion process. $J$. biol. Chem. 257, 6788-6795.

Odin, P. (1987) Chemical, immunochemical and immunohistochemical characterization of the cell adhesion molecule cellCAM 105. In Comprehensive Summaries of Uppsala Dissertations from the Faculty of Medicine No.98. Almqvist \& Wiksell International, Stockholm.

Odin, P. \& Öbrink, B. (1986) Dynamic expression of the cell adhesion molecule cell-CAM 105 in fetal and regenerating liver. Expl Cell Res. 164, 103-114.

Odin, P. \& Öbrink, B. (1987) Quantitative determination of the organ distribution of the cell adhesion molecule cellCAM 105 by radioimmunoassay. Expl Cell Res. 171, 1-15.

Odin, P. \& Obrink, B. (1988) The cell-surface expression of the cell adhesion molecule cellCAM 105 in rat fetal tissues and regenerating liver. Expl Cell Res. 179, 89-103.

Odin, P., Tingström, A. \& Öbrink, B. (1986) Chemical characterization of cell-CAM 105, a cell-adhesion molecule isolated from rat liver membranes. Biochem. J. 236, 559-568.

Odin, P., Asplund, M., Busch, C. \& Öbrink, B. (1988) Immunohistochemical localization of cellCAM 105 in rat tissues. Appearance in epithelia, platelets and granulocytes. J. Histochem. Cytochem. 36, 729-739.

O'Malley, B.W. \& Means, A.R. (1974) Female steroid hormones and target cell nuclei. Science, $N Y \mathbf{1 8 3}$, $610-620$.

Psychoyos, A. (1986) Uterine receptivity for nidation. Annls N.Y. Acad. Sci. 476, 36-42.

Psychoyos, A. \& Martel, D. (1985) Embryo-endometrial interactions at implantation. In Implantation of the Human Embryo, pp. 195-218. Eds R. G. Edwards, J. M. Purdy \& P. C. Steptoe. Academic Press, London.

Rogers, P.A.W. \& Gannon, B.J. (1981) The vascular and microvascular anatomy of the rat uterus during oestrus cycle. Aust. J. exp. Biol. Med. Sci. 59, $667-679$.

Schlafke, S. \& Enders, A.C. (1975) Cellular basis of interaction between trophoblast and uterus at implantation. Biol. Reprod. 12, 41-65.

Shaikh, A.A. (1971) Estrone and estradiol levels in the ovarian venous blood from rats during the estrus cycle and pregnancy. Biol. Reprod. 5, 297-307.

Surani, M.A.H. (1977) Cellular and molecular approaches to blastocyst uterine interactions at implantation. In Development in Mammals, vol. 1, pp. 245-305. Ed. M. H. Johnson. Elsevier/NorthHolland, Amsterdam.

Svalander, P.C., Odin, P., Nilsson, B.O. \& Öbrink, B. (1987) Trophectoderm surface expression of the cell adhesion molecule cell-CAM 105 on rat blastocysts. Development 100, 653-660.

Wood, G.S. \& Warnke, R. (1981) Suppression of endogenous avidin-binding activity in tissues and its relevance to biotin-avidin detection systems. $J$. Histochem. Cytochem. 29, $1196-1204$.

Received 21 April 1989 